\section{The Sweet Smell of Successful Cloning}

In the science fiction farce "Sleeper", Woody Allen finds himself posing as a scientist confronted with the daunting task of cloning a dead dictator from his disembodied nose. Although this technique still eludes modern science, we have come one step closer with the recent generation of a fertile mouse clone from the genetic material in a single olfactory neuron.

Scientists have on numerous occasions used nuclear transfer, wherein a somatic cell nucleus is transplanted into an enucleated germ cell, for the generation of cloned organisms. Up until now, researchers had not successfully applied this technique to postmitotic cells, such as neurons, that have irreversibly exited the cell cycle, leading to speculation that some manner of permanent DNA modification in these cells may be the obstacle to cloning.

Olfactory sensory neurons (OSNs) seem to pose a particular challenge; in the mouse, there are an estimated two million cells in the olfactory epithelium, each of which expresses only one of 1,500 different olfactory receptor genes. One prevailing model suggests that this receptor selection process may be governed by DNA recombination, in a manner similar to the mechanism used by lymphocytes in the generation of unique immune peptides.

Now, for the first time, a collaboration between Rudolf Jaenisch's group at the Massachusetts Institute of Technology (Cambridge, MA) and Richard Axel's team at Columbia University (New York, NY) has led to the successful generation of fertile, healthy mouse clones from transplanted OSN nuclei (Nature, 4 March).

The researchers created three embryonic stem (ES) cell lines by extracting nuclei from mature mouse OSNs and transplanting them into wild-type oocytes. They used these ES lines to generate viable embryos, many of which successfully matured into apparently normal, healthy, and fertile adult mice.

Because the nuclei were derived from individual OSNs, one might expect that the clones would only express the olfacto- ry receptor produced in this 'parent' cell. However, in situ hybridization and RTPCR confirmed wild-type expression of a broad range of receptors. Follow-up experiments using clones derived from cells of a specific olfactory lineage (P2) also indicated that there is no apparent impediment to the expression of the full range of olfactory receptors.

This work thus presents two important developments, demonstrating the first successful cloning from postmitotic nuclei, and strongly indicating that gene regulation, rather than actual physical DNA rearrangement, governs the ultimate selection of odorant receptor expression. - Michael Eisenstein

\section{A Neuron Is Worth a Thousand Words}

A new study with monkeys niay yield new insight into the means by which visual memories are managed and stored.

In the brain, the hippocampus has long been recognized as essential for the formation and retention of memories, although little is understood regarding how this information is managed at the cellular level. Sam Deadwyler and his colleagues at the Wake Forest University School of Medicine (Winston-Salem, NC) combined behavioral studies with physiological monitoring to try to address this intriguing question (Proc. Natl. Acad. Sci. USA, 2 March).

In a series of trials, the researcher presented rhesus monkeys (Macaca mulatta) with a series of 'clip art' images, followed by a delay, and then the opportunity to select matching images from a variety of alternatives. Some of the monkeys were also connected with electrodes that probed individual hippocampal neurons, allowing the researchers to track the response of different cells to different trial phases.

Deadwyler's team identified several neurons that seemed to provide temporal parameters for the trial process and that underwent specific stimulation by the image presentation, delay, or matching phases. These formed the majority of the cells screened, but the researchers also identified another very interesting subgroup of neurons called 'category' cells that fired only in response to specific visual components from the individual images.

In subsequent trials to better characterize these cells, researchers subtly morphed in different ways an image that stimulated a specific response, with the monkey expected to pick the best 'match'. Individual monkeys often showed different preferences: a sample image of a brown baboon might elicit a match response for either a gray dog or a brown pumpkin, depending on whether the animal perceived the match in the shape or the color, with animals apparently preferentially characterizing the images in a fashion very similar to that used by these category cells. Even over repeated trials, these selection preferences remained highly consistent.

In general, category cells tended to fire most frequently in trials with smaller numbers of 'distractor' images, wherein the monkey picked correctly; as the number of distractor images increased, the frequency with which category cells fired in response to wrong matches increased as well, suggesting that with larger numbers of visual stimuli, the chance increased of a false match that fit at least one trigger category.

The authors suggest that this categorization process may represent a more efficient way to process complex image information, rather than trying to encode every aspect of a visual memory. Like humans, individual monkeys seem to prioritize different aspects of a remembered image, although each type of categorization could potentially be of equal utility. Such categorization has been observed in other brain structures, and could be an important tool for encoding and recall of memories.

- M.E. 


\section{News Updates}

\section{Mice Disprove Plague-HIV Hypothesis}

A popular explanation for the origin of a mutation that protects against HIV infection is off base, according to a new study by researchers at The Scripps Research Institute (La Jolla, CA). Individuals with a mutant form of CCR5, which along with CD4 is needed for HIV to gain entry into a cell, do not become infected by HIV. The prevalence of this mutation-the allele frequency reaches $20 \%$ in Scandinavia-suggests that a powerful force, such as a lethal disease, selected for its spread. Because the mutation arose about 800 years ago, around the time of the Black Death in Europe, some have proposed that it protected carriers against bubonic plague. To test this hypothesis, Donald E. Mosier and his colleagues inoculated wild-type and CCR5-deficient mice with lethal doses of Yersinia pestis, the causative agent of plague. They found no significant differences in susceptibility or survival times between these two groups of animals (Nature, 12 February). These results suggest that, unless Yersinia pathogenesis differs substantially in mice and humans, the Black Death is not responsible for the high prevalence of the CCR5 mutation. Ongoing work by other groups suggests that smallpox may be an alternate explanation.

\section{Antibiotic Helps Rats Recover from Spinal Cord Injury}

Treatment with a common antibiotic following spinal cord injury (SCI) may substantially reduce the lasting effects, according to a study in rats. The long-term damage associated with SCI results from the initial mechanical injury, coupled with secondary physiological events that result in tissue loss. Minocycline is a proven neuroprotector; it inhibits the release of mitochondrial cytochrome $c$, a protein that initiates a cascade of events that lead to cell death and tissue loss. To test the drug's effects on SCI outcome, Yang D. Tang of Brigham and Women's Hospital and Children's Hospital of Boston and his colleagues produced contusion injuries to the spinal cords of female rats. One hour after injury, they administered $90 \mathrm{mg} / \mathrm{kg}$ minocycline i.p. followed by 45 $\mathrm{mg} / \mathrm{kg}$ injections every 12 hours for 5 days (Proc. Natl. Acad. Sci. USA, 2 March). All rats showed complete hindlimb paralysis the day after SCI, but over the course of 4 weeks rats that received minocycline injections recovered motor coordination and hindlimb reflexes to a significantly greater extent than the controls, which received saline injections. Histopathology suggested that the antibiotic-treated animals experienced reduced tissue loss. Given minocycline's proven safety record, it may someday become a routine treatment for SCI patients.

\section{To Improve Sunscreen-Just Add DNA}

Applying sunscreen that contains DNA fragments before sunbathing may reduce your risk of developing skin cancer, according to research in mice. During sun exposure, UV rays are absorbed by the skin, resulting in the production of mutagenic photoproducts that damage DNA and cause cancer. Sunscreens passively block the absorption of UV rays but do not help cells repair sun damage. A research team led by Barbara A. Gilchrest of the Boston University School of Medicine (Boston, MA) applied a solution containing DNA fragments called thymidine dinucleotide (dTT) to the skin of two strains of hairless mice: wild-type (WT) and a DNA repair-deficient mouse model of the rare disease xeroderma pigmentosum (XP). Over the course of 75 days, Gilchrest's team exposed the mice to UV light, with intermittent applications of the dTT solution (Proc. Natl. Acad. Sci. USA, 16 March). They found that topical application of dTT induced mechanisms of DNA repair and reduced the mutation rate and tumor incidence not only in the strains proficient in DNA repair but in those deficient in DNA repair as well. By week 24, mice treated with dTT had a much lower incidence of skin tumors (22\% WT and 50\% XP) than controls that did not receive dTT treatments (88\% WT and $100 \%$ $\mathrm{XP}$ ). If a similar solution proves safe and effective in humans, DNA-containing sunscreens may someday find their way onto the pharmacy shelves. 